\title{
High-resolution resonant and nonresonant fiber-scanning confocal microscope
}

\author{
Benno H. W. Hendriks, Walter C. J. Bierhoff, Jeroen J. L. Horikx, Adrien E. Desjardins, Cees A. Hezemans, \\ Gert W. 't Hooft, Gerald W. Lucassen, and Nenad Mihajlovic \\ Philips Research, Minimally Invasive Healthcare Department, Eindhoven 5656 AE Eindhoven, The Netherlands
}

\begin{abstract}
We present a novel, hand-held microscope probe for acquiring confocal images of biological tissue. This probe generates images by scanning a fiber-lens combination with a miniature electromagnetic actuator, which allows it to be operated in resonant and nonresonant scanning modes. In the resonant scanning mode, a circular field of view with a diameter of $190 \mu \mathrm{m}$ and an angular frequency of $127 \mathrm{~Hz}$ can be achieved. In the nonresonant scanning mode, a maximum field of view with a width of $69 \mu \mathrm{m}$ can be achieved. The measured transverse and axial resolutions are 0.60 and $7.4 \mu \mathrm{m}$, respectively. Images of biological tissue acquired in the resonant mode are presented, which demonstrate its potential for real-time tissue differentiation. With an outer diameter of 3 $\mathrm{mm}$, the microscope probe could be utilized to visualize cellular microstructures in vivo across a broad range of minimally-invasive procedures. $\odot 2011$ Society of Photo-Optical Instrumentation Engineers (SPIE). [DOI: 10.1117/1.3534781]
\end{abstract}

Keywords: scanning microscopy; confocal optics; actuators, microscopy; fiber optic applications; biomedical optics.

Paper 10504R received Sep. 15, 2010; revised manuscript received Dec. 3, 2010; accepted for publication Dec. 15, 2010; published online Feb. 7, 2011.

\section{Introduction}

Microscopic imaging of living tissue in vivo could allow for real-time disease diagnosis. ${ }^{1}$ Various approaches have been investigated to develop miniature microscopes that are compatible with minimally invasive procedures. Most of these approaches involve either single-fibers or fiber-bundles to transmit and receive light [as reviewed in Refs. 2 and 3)]. Important design criteria are the spatial resolution, the field of view (FOV), as well as the contrast that can be achieved. Designs based on coherent fiber bundles have many advantages including the potential for high levels of miniaturization and mechanical flexibility, ${ }^{4-6}$ but are limited in resolution by the diameter and spacing of the fibers. With some designs, Fresnel reflection of illumination light from the distal ends of the fibers can impose limitations on the dynamic range; fiber autofluorescence can also have confounding effects. Single-fiber solutions require an actuation method to move the fiber tip at the distal end of the probe. Methods employing piezomotors, ${ }^{7,8}$ microelectromechanical systems (MEMS), ${ }^{9,10}$ and tuning-forks ${ }^{11}$ have been investigated. In most of these approaches, the objective lens system in front of the fiber distal end is not actuated, which results in constraints on the achievable numerical aperture (NA) and the FOV of the scanner. ${ }^{12,13}$

Different optical imaging modalities can be employed in a single-fiber optic scanner such as confocal reflectance, ${ }^{13}$ confocal fluorescence, ${ }^{14}$ two-photon fluorescence, ${ }^{15,16}$ and optical coherence tomography. ${ }^{17,18}$ Most single-fiber scanning microscopes that have been previously demonstrated operate in resonant modes; as such, they may not be ideal for applications requiring longer acquisition times such as two-photon microscopy and Raman spectroscopy.

Address all correspondence to: Benno Hendriks, Minimally Invasive Healthcare, Philips Research, HighTechCampus 34 - Eindhoven, Noord-Brabant 5656 AE Netherlands; Tel. 31402747832; E-mail: benno.hendriks@philips.com.
In this paper, we present a single-lens, high resolution, electromagnetically-controlled confocal fiber-scanning microscope with an outer diameter of $3.0 \mathrm{~mm}$, that can be operated in resonant and nonresonant modes. As a preliminary indication of the capability of this microscope to perform tissue differentiation, we present images obtained from tissues with confocal reflectance with and without polarization gating.

\section{Materials and Methods}

\subsection{Optical Design}

The scanner design involves a single optical fiber with a flexible distal end. The position of the distal end of the fiber is controlled by two sets of electromagnetic coils that allow for deflections in orthogonal directions transverse to the probe axis. To focus the light beam delivered from the fiber, an objective lens system is required. This objective lens system in front of the distal end of the fiber could either be attached to the fiber or mounted on the housing of the scanner. When the lens system is mounted to the housing, the FOV that can be achieved with the given stroke of the fiber tip $P_{\text {fiber }}$ scales with the ratio of NA of the objective lens system, $N A_{o b j}$, and the NA of the exit beam, $N A_{\text {fiber }}$. With $N A_{o b j}$ $=0.65$ and $N A_{\text {fiber }} \sim 0.1$, a stroke of $P_{\text {fiber }}=1.3 \mathrm{~mm}$ is required in order to acheive a FOV of $200 \mu \mathrm{m}$. The large ratio between the stroke distance and the FOV imposes severe constraints on the FOV for small diameter sized fiber scanners. Another drawback of this approach is that complicated objective lens systems are required to allow for illumination of the lens to be performed at different angles while maintaining constant resolution. ${ }^{12,13,19}$ In the approach taken in this study, the objective lens system is mounted to the moveable part of the fiber at a fixed distance from the fiber tip. In this way, the FOV is equal to the lateral stroke of the fiber. Furthermore, the beam enters the lens on-axis, allowing for the use of a single-lens with a high NA. ${ }^{20}$ The objective

1083-3668/2011/16(2)/026007/8/\$25.00 (C) 2011 SPIE 
(a)
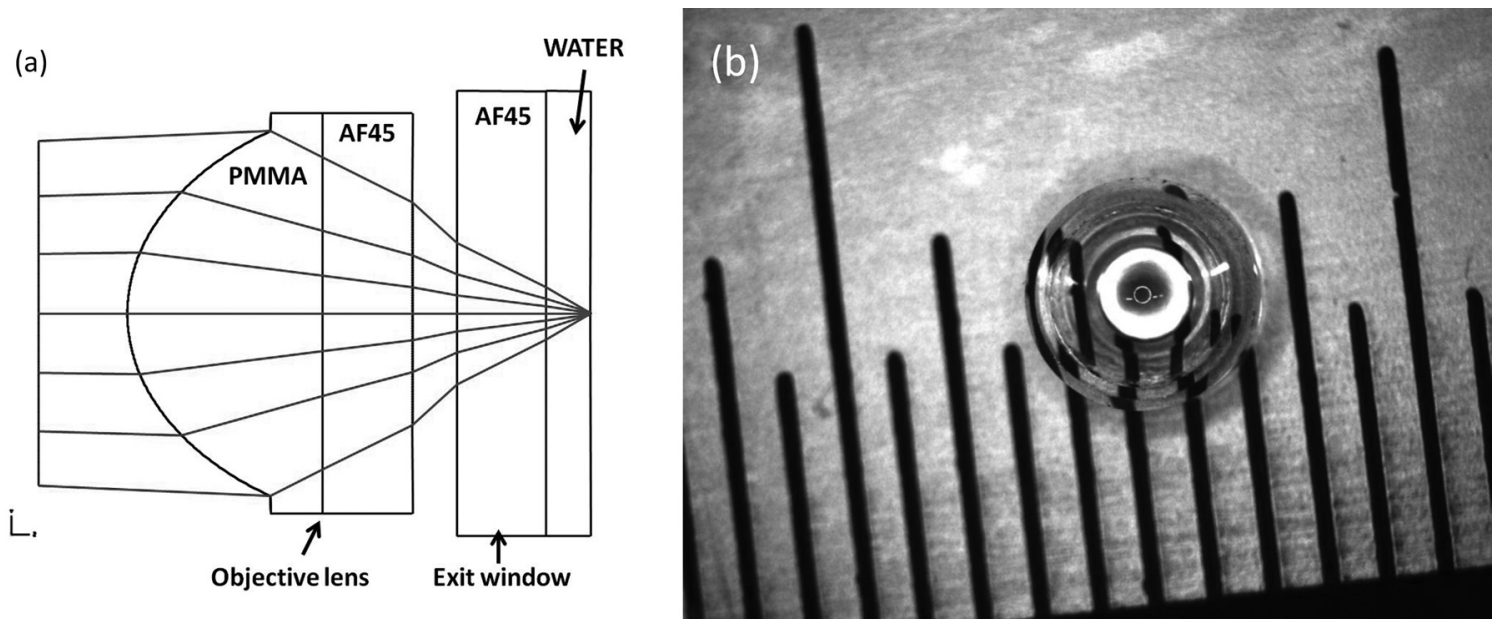

Fig. 1 The single plano-aspherical objective lens: (a) optical design layout and (b) a photograph of a manufactured objective lens (the line separation of the ruler is $0.5 \mathrm{~mm}$ )

lens system in our scanner is plano-aspherical, constructed from poly(methylmethacrylate) (PMMA) (refractive index $n=1.492$ and Abbe number $V=57.4$ ), and mounted on a $0.2 \mathrm{~mm}$ thick AF45 Schott glass plate with a design wavelength of $780 \mathrm{~nm}$ (Fig. 1). For fast prototyping, the aspheric lens was directly diamond turned on a high precision lathe. For this lens, $N A_{o b j}$ $=0.68$; the entrance pupil was $0.82 \mathrm{~mm}$ and the focal length was $0.68 \mathrm{~mm}$. The total on-axis thickness of the lens was 0.65 $\mathrm{mm}$. The objective lens was positioned at a distance of $10 \mathrm{~mm}$ from the distal end of the fiber. The air gap between the objective and the proximal end of the exit glass window was $0.1 \mathrm{~mm}$. The exit window had a thickness of $0.2 \mathrm{~mm}$ and was constructed of AF45 Schott glass. The objective lens focused the exit beam of the fiber at a distance of $0.1 \mathrm{~mm}$ beyond the distal end of the exit window that was optimized for immersion in a water-like environment $(n=1.33)$.

In order to be flexible for different imaging modalities, especially for modalities involving short pulses such as two-photon imaging, chromatic aberration introduced by the objective lens must be low. ${ }^{21,22}$ With our objective lens system, chromatic aberration results in a time shift $\Delta T$ between the marginal ray and the principle ray. In order to maintain a short pulse width, the time shift must be smaller than the pulse width $\Delta \tau$. According to Bor, ${ }^{22}$ the time shift is given by

$$
|\Delta T|=\left|\frac{N A_{o b j}^{2} \lambda f}{2 c(n-1)} \frac{d n}{d \lambda}\right|=\left|\frac{N A_{o b j}^{2} f \lambda}{2 c\left(\lambda_{F}-\lambda_{C}\right) V}\right|,
$$

where $\lambda$ is the wavelength, $N A_{o b j}$ is the numerical aperture objective, $c$ is the speed of light, $n$ is the refractive index, $f$ the focal length of the lens, and $V$ is the Abbe number of the lens material. The two wavelengths $\lambda_{F}$ and $\lambda_{C}$ are the Fraunhofer F- and C-spectral lines, given by $\lambda_{F}=486.13 \mathrm{~nm}$ and $\lambda_{C}=656.27 \mathrm{~nm}$. For the objective lens system in our microscope, we find that $\Delta T$ $=42 \mathrm{fs}$, which is smaller than pulse widths of approximately $100 \mathrm{fs}$ that are typical for two-photon microscopy applications.

\subsection{Mechanical Design}

A drawing of the mechanical construction as well as a photograph of the fiber scanning microscope is shown in Fig. 2. It consists of the cylindrical fiber housing, which is constructed from a stainless steel cylindrical tube with an inner diameter of $0.25 \mathrm{~mm}$ and outer diameter of $0.5 \mathrm{~mm}$. The proximal end of this tube is rigidly connected to the microscope housing; the distal part of this tube can move freely. The length of the fiber housing is $40 \mathrm{~mm}$. We note that the mechanical properties of the scanner are determined by the fiber housing and not by the fiber itself. This property has the advantage that approximately the same resonant frequency can be maintained with different optical fibers. At the distal part of the fiber housing, an objective lens is mounted.

Two independently-driven driving coil pairs are fixed to the microscope housing, as shown in Fig. 2. In order to minimize the outer diameter of the microscope housing, the central axes of the coils are chosen to be perpendicular to the flux of the magnet. When a current is applied to one of the coil pairs, the magnet experiences Lorenz forces in a direction that depends on the sign of the current. By application of appropriate currents in
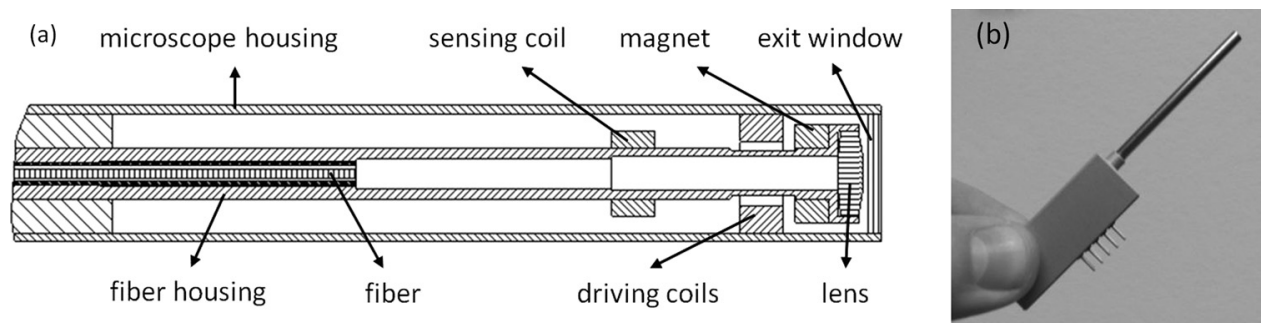

Fig. 2 (a) Schematic drawing (not to scale) and (b) photograph of the scanning fiber microscope. 


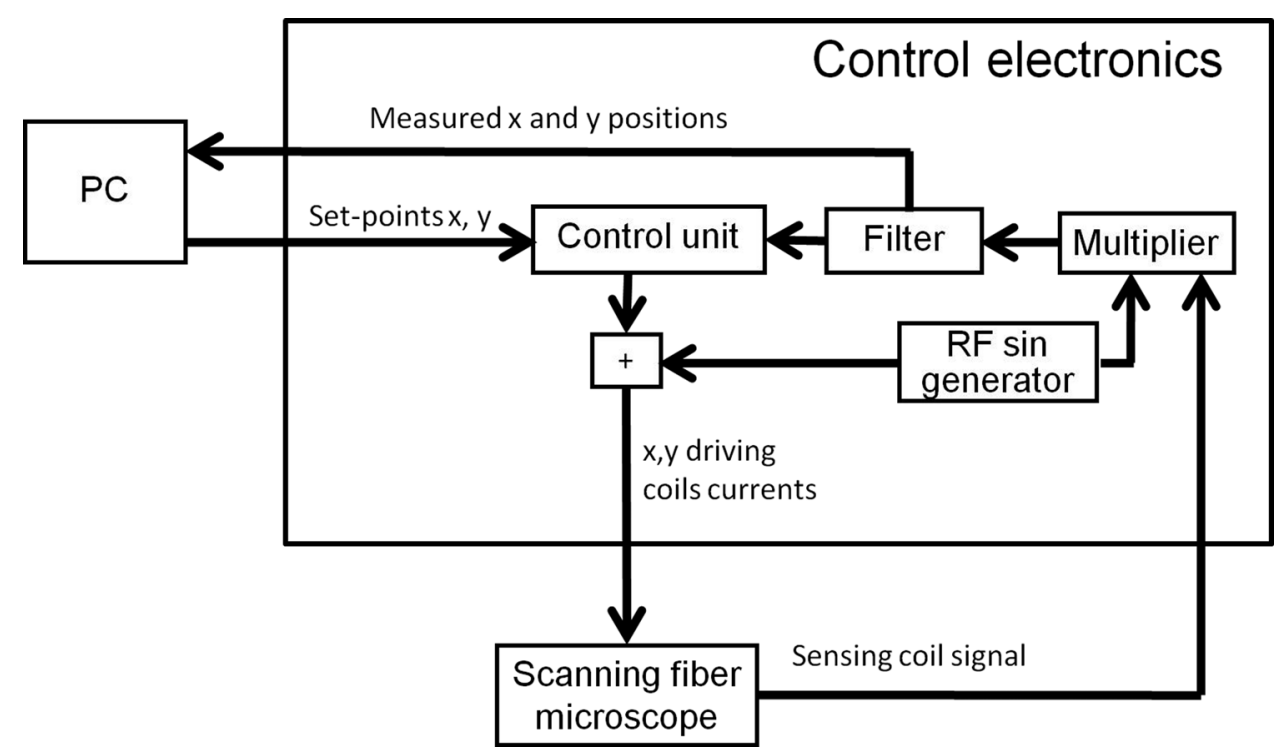

Fig. 3 Schematic diagram of the control electronics. The position information of the objective lens coming from the sensing coil is compared with the required set-points. Depending on the deviation from the required set-points the control unit adjusts the position of the lens.

the coil pairs, the fiber tip and lens can be arbitrarily positioned in $x$ - and $y$ - lateral directions within the scanning area.

A sensing coil is attached to the lens mount to measure the position of the fiber tip and lens with respect to the microscope housing. While the electromagnetic coupling between magnet and driving coils deliver the drive forces, the electromagnetic coupling between the sensing coil and driving coils delivers the position information. The position measurement method is described in detail in Sec. 2.3.

From a mechanical standpoint, the scanning part of the system can be modeled as a hollow tube with a distributed mass $m$ (lens, lens mount, magnet, and sensing coil) at the distal end of the tube. This total distributed mass is $m=55 \mathrm{mg}$. The hollow tube is fixed at the proximal end, while the distal end can move freely. Taking the shapes and materials into account, the stiffness of our system is calculated to be $k=37.5 \mathrm{~N} / \mathrm{m}$. The resonance frequency $f_{\text {res }}$, is given by

$$
f_{\text {res }}=\frac{1}{2 \pi} \sqrt{\frac{k}{m}} .
$$

Equation (4) predicts that for our microscope, $f_{\text {res }}$ is equal to $131 \mathrm{~Hz}$.

The microscopic image is formed by making a spiral movement with the distal end of the fiber. The field of view FOV res of the scanner in the resonance mode can be estimated as follows. The electromechanical coupling constant $k_{e m}$ can be computed from the design of the driving coils and the location of the magnet and is determined to be $k_{e m}=3.7 \mathrm{mN} / \mathrm{A}$. Measurements on the maximum allowed current through the driving coils such that the temperature in the coils remains below $70{ }^{\circ} \mathrm{C}$ is found to be $0.42 \mathrm{~A}$. To include a safety margin, we limited the maximum current to $I_{\max }=0.35 \mathrm{~A}$ in our system. Finally, the quality factor $Q$ (i.e., the amount of underdamping of the resonator) is designed to be larger than 50. From these parameters, the FOV which is defined by

$$
F O V^{r e s}=\frac{k_{e m} I_{\max }}{\pi b f_{\text {res }}},
$$

with the damping coefficient $b$ given by

$$
b=\frac{\sqrt{k m}}{Q}
$$

where $k$ is the stiffness, $m$ the distributed mass, and $Q$ is the quality factor, yielding $\mathrm{FOV}^{\text {res }}=3.46 \mathrm{~mm}$. For a typical required FOV of $0.2 \mathrm{~mm}$, the system has sufficient tolerance to cope for instance with manufacturing errors. This large FOV at resonant scanning shows that with nonresonant scanning, a significant FOV can still be achieved. For example, if the scanning is performed with a very low frequency, the field of view $F O V^{\text {non }}$ is then given by

$$
F O V^{n o n}=2 \frac{k_{e m} I_{\max }}{k} .
$$

For our given parameters, Eq. (7) yields a prediction of $F O V^{n o n}=69 \mu \mathrm{m}$.

\subsection{Electronic Design}

In order to estimate the position of the fiber tip in real-time during scanning, we let the current through $x$ - and $y$-coils, $I_{x}$ and $I_{y}$, consist of driving currents and sensing currents: $I_{x}=I_{d x}+I_{s x}$ and $I_{y}=I_{d y}+I_{s y}$ (see Fig. 3). The sensing currents are high-frequency sinusoidal signals with zero DC components. Their frequencies are much higher than the angular scanning frequency, so that they have a negligible effect on the lens displacement. Furthermore, the frequencies of the $I_{s x}$ and $I_{s y}$ signals differ from each other in order to independently measure positions of the lens in $x$ - and $y$-directions. The sensing currents $I_{s x}$ and $I_{s y}$ induce currents $I_{i x}$ and $I_{i y}$ in the sensing coil, respectively [see Fig. 2(a)]. The amplitude of the induced current $I_{i x}$ $\left(I_{i y}\right)$ represents the difference between the currents induced by the $x$ - $(y$-) coil pairs. When the sensing coil is at the center of the $x$ - $(y-)$ coil pairs, the induced current $I_{i x}\left(I_{i y}\right)$ is zero. Furthermore, the amplitudes of these induced currents are linearly 

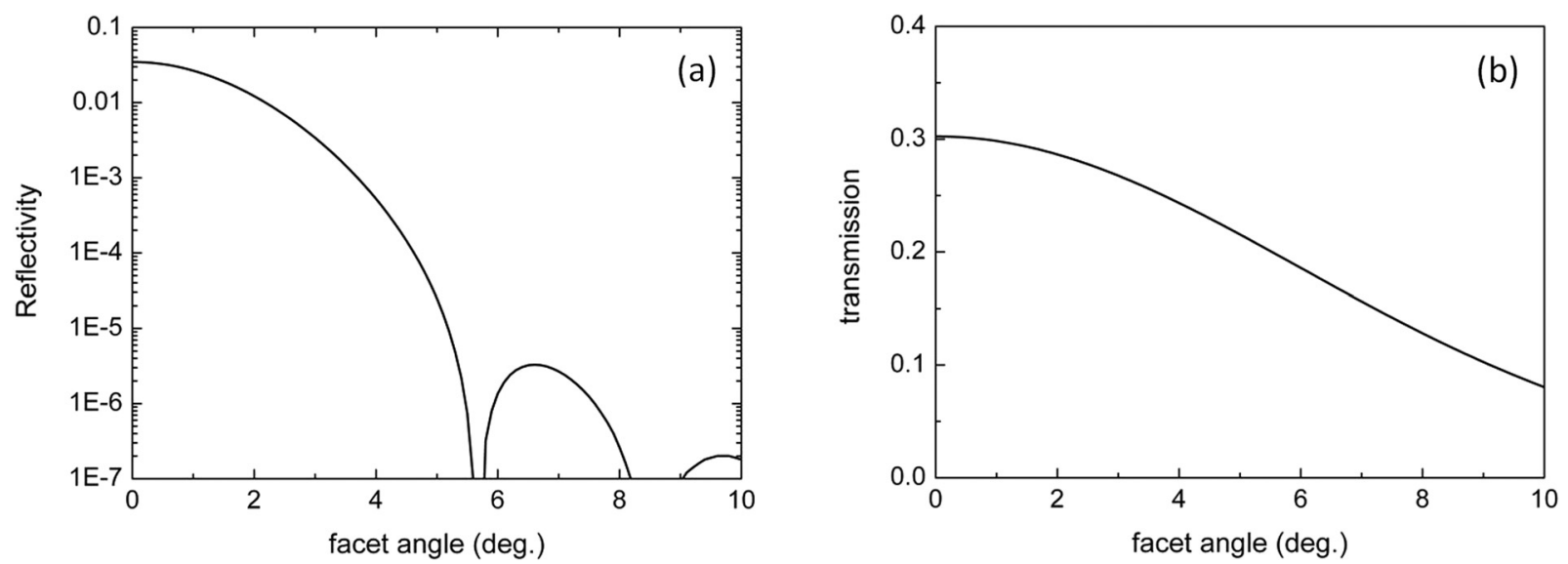

Fig. 4 (a) Computed facet reflectivity and (b) fraction of power captured by the entrance pupil of the objective as a function of polishing angle of the fiber facet in the scanner. In the computations, a step-index quartz fiber with a core radius of $2.32 \mu \mathrm{m}$ and an $N A$ of 0.13 has been assumed, with $\lambda=785 \mathrm{~nm}$. The objective lens parameters are described in Sec. 2.1.

dependent on the displacements of the measurement coil for the relevant displacements encountered in the scanner system.

In order to obtain the amplitudes of $I_{i x}$ and $I_{i y}$, the signal from the sensing coil is independently multiplied by $I_{s x}$ and $I_{s y}$, respectively, and then filtered with a low pass filter. This demodulation method (i.e., the method of synchronous detection) allows for the simultaneous detection of the $x$ - and $y$-positions of the sensing coil. Finally, a proportional-integral-derivative controller (PID controller) has been implemented in the control unit (see Fig. 3) to control the position of the lens.

\subsection{Console Optics Design}

The light source is a superluminescent light-emitting diode (EXALOS1, EXS8010-2411), coupled into single-mode fiber (SM800-5.6-125). It has a spectrum of $\sim 25 \mathrm{~nm}$ width [full width at half maximum (FWHM)], centered at $785 \mathrm{~nm}$ and a maximum output power of $5 \mathrm{~mW}$. A light source with short coherence length was chosen to prevent interference fringes that result from interference between the light reflected by the sample and the (unwanted) light reflected by the polished face of the fiber inside the scanner.

To reduce its reflectivity, the fiber face is polished at an angle. This introduces a linear phase variation over the mode reflected at the facet, greatly reducing the efficiency with which it couples back into the fiber. Also, due to refraction, the far-field pattern of the fiber mode shifts over the entrance pupil of the objective lens and the amount of light captured by the lens and focused onto the sample is diminished. The results of model calculations for a representative step-index fiber are displayed in Fig. 4. A polishing angle of $5 \mathrm{deg}$ has been selected, giving a reduction of the facet reflectivity of about three orders of magnitude, while decreasing the amount of light captured by the lens by only about one-third.

Figure 5(a) shows the optical system utilized for polarizationinsensitive detection. A polarization-independent fiber-optical circulator (OFR, OC-3-780-FC/APC3) was used to direct reflected light to a detector. For samples with relatively high reflectivity, a large-area photoreceiver (New Focus, Model 2031) is used, while for low-reflectivity samples, a more sensitive avalanche photodiode module is used (Hamamatsu, C5460-01).
Figure 5(b) shows the optical system utilized for polarizationsensitive detection. The incident light, collimated by lens $\mathrm{L}_{1}$ (part of a PAF-X-5-B FiberPort collimator module from ThorLabs), passes through a polarizing beam splitter cube (PBS, PSCLB-VR-780 from ThorLabs; extinction ratio $>1000: 1$ at the design wavelength of $780 \mathrm{~nm}$ ) and is focused into the polarization-maintaining scanner fiber (Nufern, PM780 -HP). A $\lambda / 2$ waveplate provides some control over the polarization of the light incident on the PBS; it can be rotated to maximize the amount of light incident on the sample. The $\lambda / 4$ waveplate between the PBS and the PM scanner fiber determines which polarization component of the reflected light was directed by the PBS toward the detector. When the axes of the $\lambda / 4$ waveplate are parallel to those of the PBS, only cross-polarized light is detected; light of parallel polarization is included when the waveplate is rotated (however, also the light reflected by the fiber facet will then be detected to a larger degree).

To convert the intensities measured during the spiral scanning into an image, we proceeded as follows. The image on the screen was divided into equal rectangular bins onto which the segments of the spirals were mapped. Since the angular velocity and the pitch of the spiral movement were kept constant during scanning, the measurement time for each bin was longer for the central pixels than for the outer pixels. Aside from the Polar to Cartesian map, the only image processing employed was the application of an offset and scaling to intensities.

\section{Results and Discussion}

\subsection{Mechanical Properties}

The measured frequency response functions of the scanning system in $x$ - and $y$-direction are shown in Fig. 6, revealing a resonance frequency of $127 \mathrm{~Hz}$ in the $x$-direction and $115 \mathrm{~Hz}$ in the $y$-direction. These values are in close agreement with the predicted value of $131 \mathrm{~Hz}$. The electronic control system can correct for these differences in resonant frequencies and we selected $127 \mathrm{~Hz}$ as the scanning frequency. The Q-factor in $x$-direction is 62 and in $y$-direction 47 , which also agrees well with the designed value of 50 .

With the actuator, a circular FOV with a maximum diameter of $190 \mu \mathrm{m}$ can be imaged, see Fig. 7(a). Note that this FOV is 

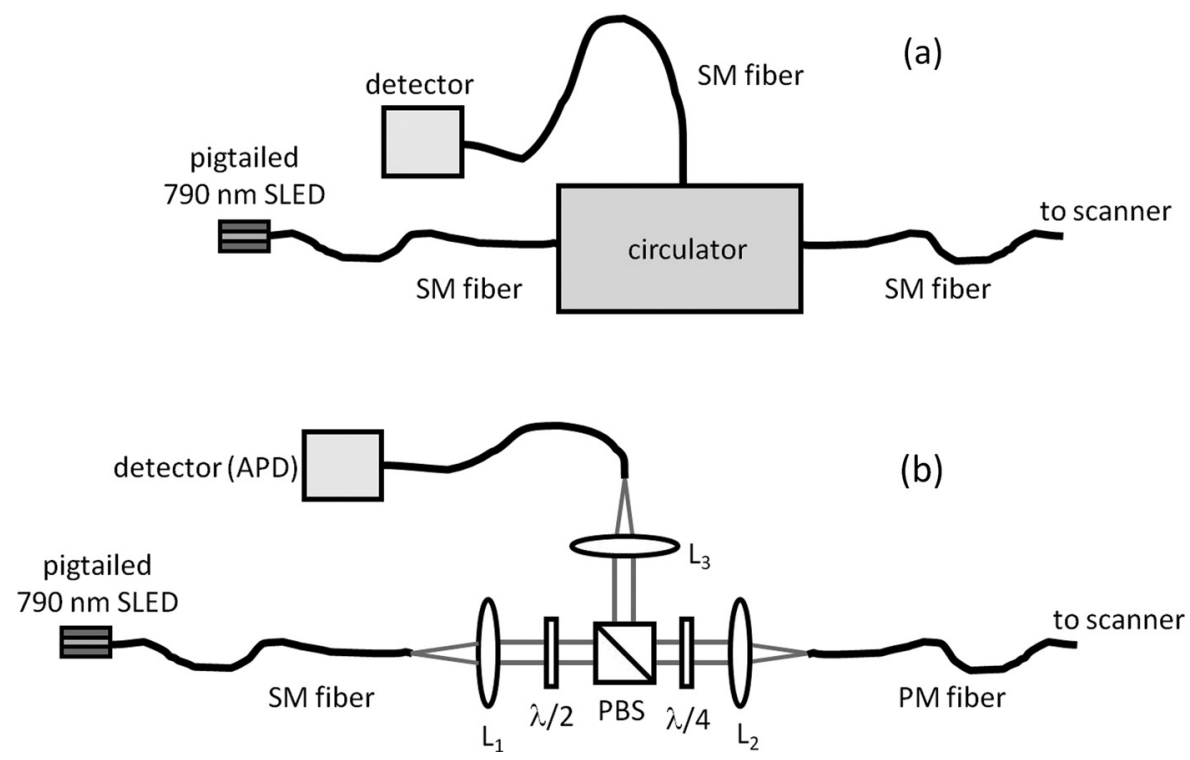

Fig. 5 Console optics: (a) single mode (SM) fibers setup for polarization-insensitive backscattered light imaging mode; (b): polarization-sensitive setup with a polarization-maintaining fiber in the scanner.

determined by the space available in the housing to make the stroke and not by the actuator. At a very low scanning speed (i.e., nonresonant scanning), it is possible to scan an area with a diameter of $69 \mu \mathrm{m}$ as shown in Fig. 7(b). The nonresonant scan width agrees very well with our predicted value.

\subsection{Optical Resolution}

The resolution that can be obtained by the fiber scanner system depends on the objective lens pupil filling. When we overfill the pupil of the objective lens, the spot width is given by the Airy distribution having a FWHM of the intensity in the lateral direction of $\Delta x=0.51 \lambda / N A$, under the assumption that aberrations are negligible. At a center wavelength of $780 \mathrm{~nm}$ and NA of 0.68 , that formula predicts a transverse resolution $\Delta x$ of $0.59 \mu \mathrm{m}$. In practice, however, the pupil filling will exhibit a distribution that is approximately Gaussian with a rim intensity that depends on the choice of the fiber used in the scanner and the distance of the fiber end to the objective lens.

(a)
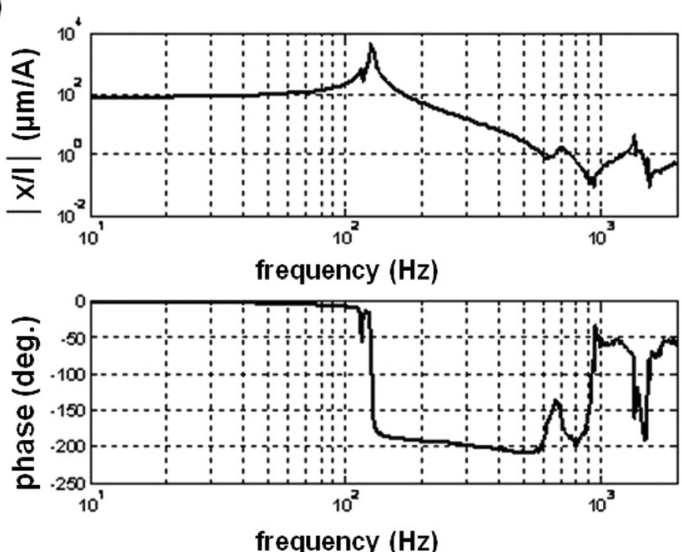

The lateral resolution was measured with a reflective edge in the focal plane of the objective. Under the assumption that the spot has a Gaussian spatial distribution, the FWHM of the lateral distribution can be approximated by 0.92 times the 10-90\% edge width. ${ }^{23,24}$ Figure 8 (a) shows that this FWHM is $0.60 \mu \mathrm{m}$ at a wavelength of $780 \mathrm{~nm}$. To experimentally determine the axial resolution, the detector intensity was measured when moving a plane mirror surface through the object focal plane. As shown in Fig. 8(b), the measured FWHM was $7.4 \mu \mathrm{m}$.

\subsection{Imaging}

The image contrast that can be achieved depends on the optical throughput of the fiber-objective system, as well as on the optical modality employed to generate optical contrast in the tissue. As a fraction of the light intensity that was coupled into fiber from the source, the light intensity that reached the focal point was measured to be 0.34 for polarization-insensitive detection. This throughput is consistent with the calculated value shown

(b)

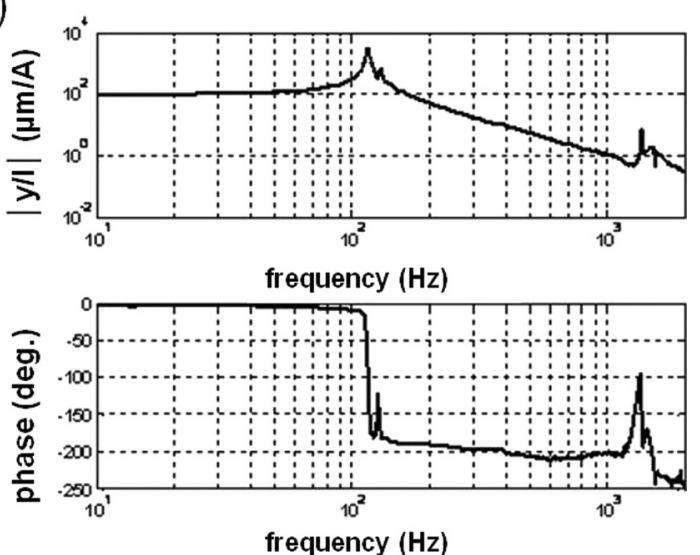

Fig. 6 Measured frequency response functions (position divided by the applied current) of the electromagnetic actuator in (a) $x$ - and (b) $y$-scanning direction. 

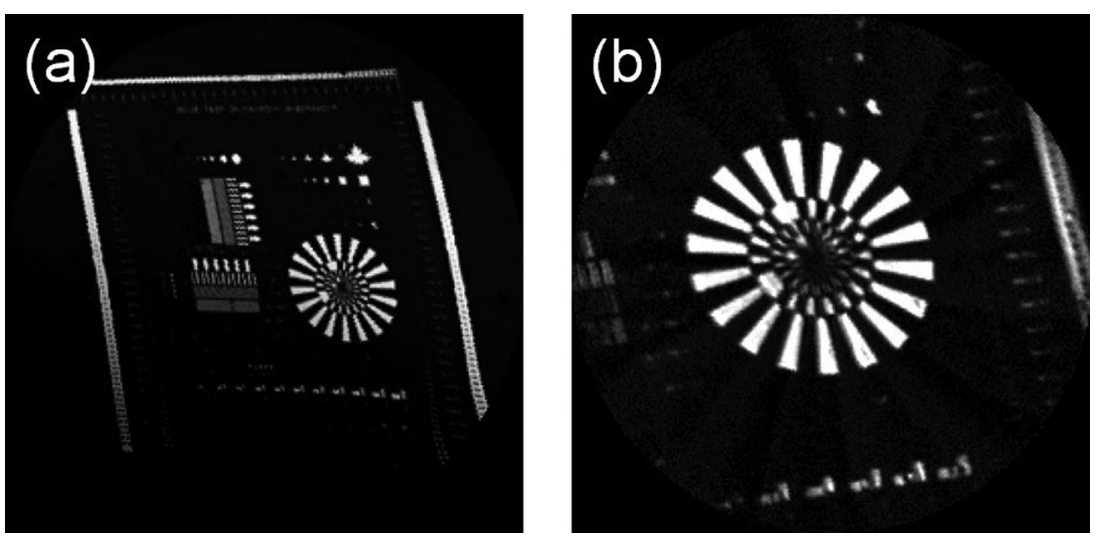

Fig. 7 Images of a Richardson microscope test slide (Ref. 25) made with the scanning fiber microscope at various scanning speeds: FOV of (a) $190 \mu \mathrm{m}$ obtained in $1.2 \mathrm{~s}$ and (b) $69 \mu \mathrm{m}$ obtained in $15 \mathrm{~s}$.

in Fig. 4(b). It is determined by the fiber numerical aperture, the fiber-objective lens distance, the entrance pupil of the objective, and the losses in the objective lens system. In order to allow for the possibility of integrating different types of fibers with smaller numerical apertures than that of the polarization insensitive fiber used in this study, there was substantial overfilling of the objective.

To demonstrate the potential of the scanner, we present several images of tissue samples for the polarization-insensitive and polarization-sensitive detection.

In Fig. 9, images taken of ex vivo tissues with the scanning fiber microscope with polarization-insensitive detection are shown. Figure 9(a) shows a zoomed-in image of the Richardson microscope test slide ${ }^{25}$ with a $50 \mu \mathrm{m}$ FOV. Figure 9(b) shows an image of an ex vivo pig bronchus wall with $120 \mu \mathrm{m}$ FOV, in which contrast likely derived primarily from of ex vivo tissues collagen fibers.

In Fig. 10, images taken of ex vivo tissue with the scanning fiber microscope with polarization-sensitive detection are shown. Each image had a FOV of $190 \mu \mathrm{m}$ and was constructed with 150 spirals so that the acquisition time was $1.2 \mathrm{~s}$. Figure 10(a) shows an image of a rat skeletal muscle with the microscope positioned in a rigid stand during the acquisition, in which striations are clearly apparent. In Fig. 10(b), the same tissue re- gion shown in Fig. 10(a) was targeted, but in these cases the scanner was held by hand during acquisition without a rigid stand. The striations remained visible, which demonstrates that our microscope is relatively insensitive to the confounding effects of hand motion. Figure 10(c) shows an image of a rat hair with the texture of the outermost layer of the hair shaft (cuticle layer) clearly visible. Figure 10(d) shows an image of the inner surface of a mouse artery. In this image, the regions of high reflectance likely correspond to collagen fibers. Figure 10(e) shows a similar image of the artery wall with imaging performed at a different depth. In this image, fine structures that may correspond to elastin fibers are apparent. Figure 10(f) shows an image of a rat liver treated with $5 \%$ acetic acid, with areas of the nuclei of liver cells likely manifesting as regions of high reflectivity.

\subsection{Discussion}

In the current design, the outer diameter of the scanner is $3 \mathrm{~mm}$. We estimate that downscaling the scanner to approximately $2 \mathrm{~mm}$ can be done with the same performance. Smaller dimensions become challenging, however, because manufacturing a small sized electromagnetic motor that can deliver a sufficient force is difficult at these scales. Furthermore, 3-dimensional imaging can be realized in the scanner by adding
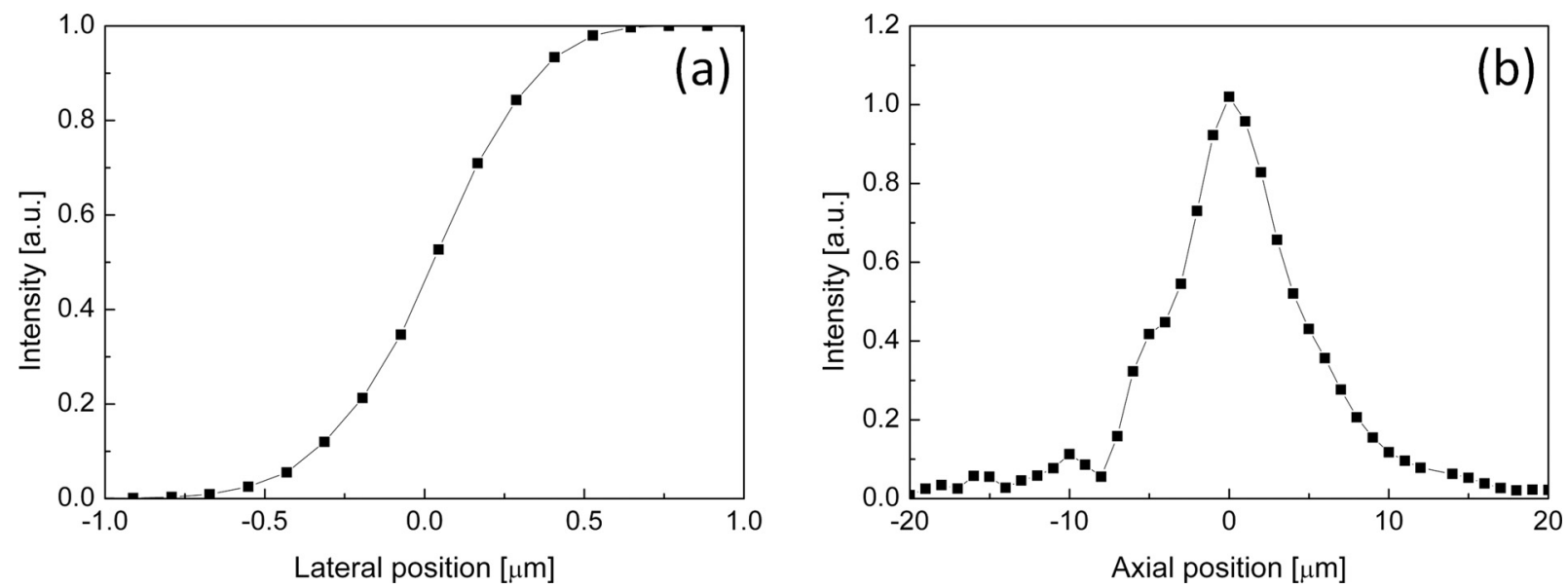

Fig. 8 The measured intensity on the detector when (a) moving an edge of a mirror in the lateral direction along the focal plane (i.e., edge response) and (b) moving a plane mirror surface through the object focal plane of the fiber scanner. 

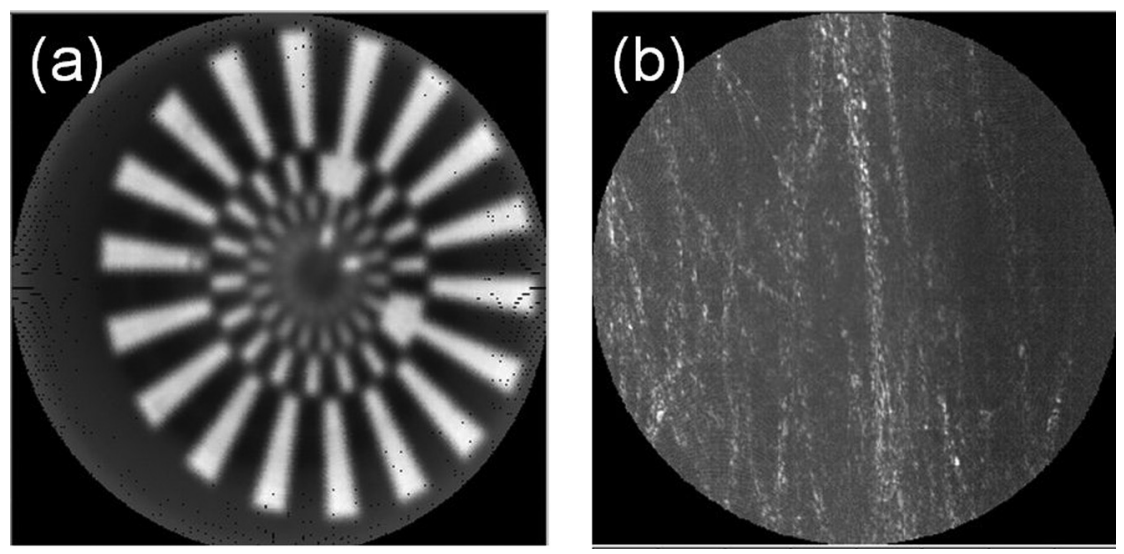

Fig. 9 Images acquired with the scanning fiber microscope and using the backscattered light imaging modality [see Fig. 4(a)]: (a) an image of the Richardson microscope test slide with $50 \mu \mathrm{m} \mathrm{FOV,} \mathrm{(b)} \mathrm{pig} \mathrm{bronchus} \mathrm{wall} \mathrm{with} 120 \mu \mathrm{m}$ FOV where collagen present in the inner bronchus wall can be seen.

an actuator in the connection between the fiber housing and the microscope housing. Most of the fiber scanners reported in literature have scanning speeds above 2.5 frames per second (fps). Our current scanner design achieves a frame rate of 1 fps, which we showed to be sufficient to obtain well-resolved microscopic images when the scanner is held by hand while in contact with the tissue. Higher frame rates with the same spatial resolution could be achieved with our design (for instance by reducing the length of the fiber housing), but this would limit the FOV that can be achieved in the nonresonant mode. The current optical design, in which the lens is attached to the fiber, allows high numerical apertures to be realized. Numerical apertures of 0.85 for single objectives smaller than $1 \mathrm{~mm}$ pupil diameter have been reported in Ref. 20. The achievable numerical aperture for fixed entrance pupil diameter is limited by the required free working distance. When the objective lens is attached to the fiber, an additional exit window is required, which reduces the free working distance by approximately $75 \mu \mathrm{m}$ compared to fiber scanners in which the objective fixed to the microscope housing. The resolution achieved across the FOV is determined
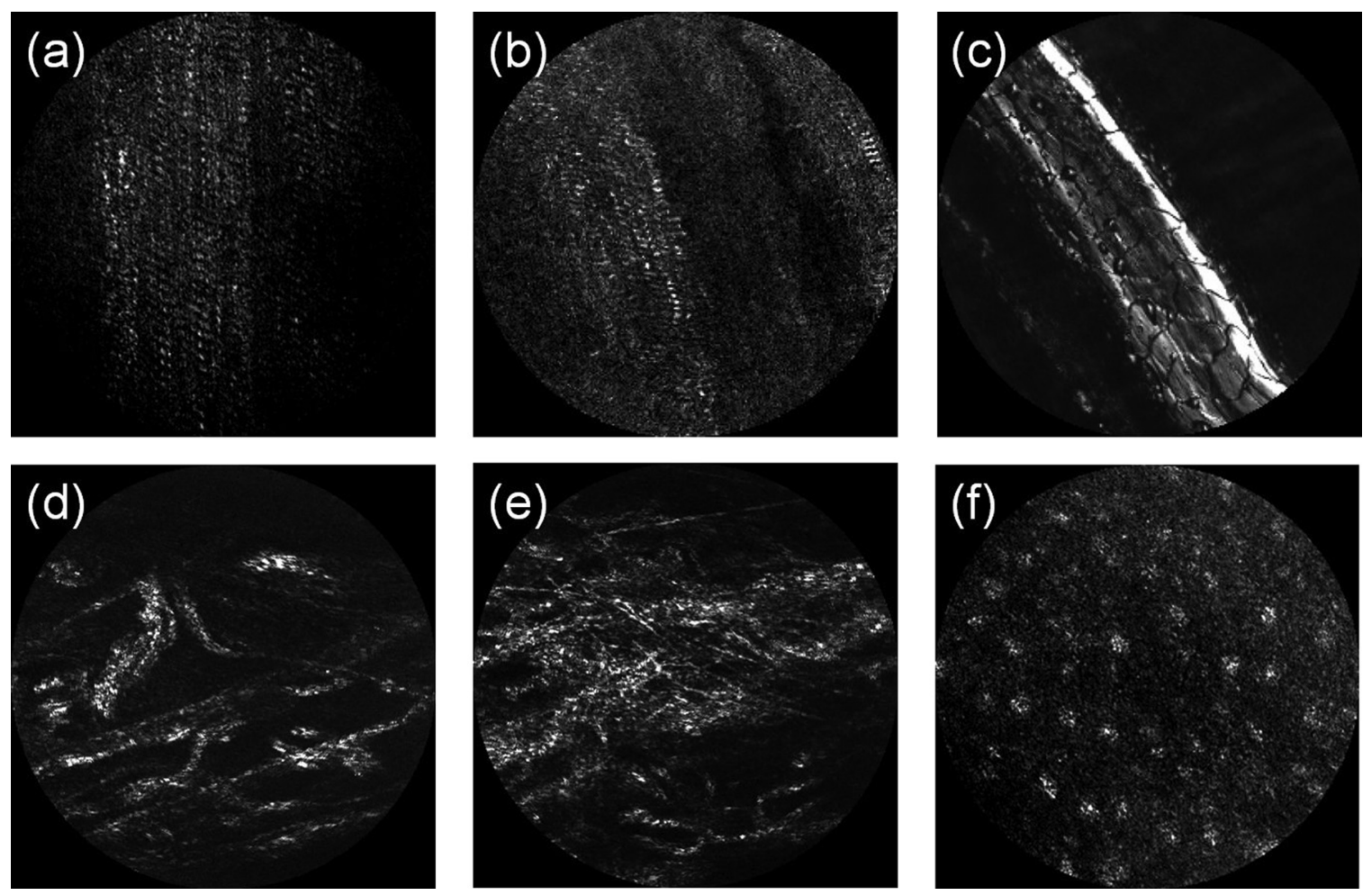

Fig. 10 Images acquired with the scanning fiber microscope using the polarization-sensitive reflectance optical modality [see Fig. 4(b)] with a FOV of $190 \mu \mathrm{m}$; (a) rat skeletal muscle with microscope in holder during image acquisition, (b) same as (a) but with microscope held with hand during image acquisition, (c) image of a rat hair, (d) image of a mouse artery where the white structures are likely the collagen fibers present in the artery wall, (e) image of a mouse artery where the white structures are likely elastin fibers present in the artery and (f) image of a rat liver treated with $5 \%$ acetic acid where the white areas are likely the nuclei of the liver cells. 
by the numerical aperture as well as the lens aberrations. For the objective attached to the fiber, the lens system is used only onaxis, resulting in a constant resolution throughout the FOV. This allows for a simple lens design, as compared to lens systems that are fixed to the microscope housing.

The images shown in Fig. 10 illustrate the potential use of the scanner in relevant biological applications. For instance the images in Figs. 10(d) and 10(e) show that certain structures in the blood vessels that are known to play a role in cardiovascular disease (see for instance Ref. 26) can be visualized, while Fig. 10 (f) reveals structures that are relevant to determine diseased tissues in the field of oncology.

\section{Conclusions}

The design and implementation of a high-resolution fiberscanning confocal microscope with an outer diameter of $3 \mathrm{~mm}$ were presented. The images obtained from biological tissue demonstrate that microscopic-level imaging can be achieved even with the microscope held by hand. Further studies are required to determine the potential of this microscope to provide information relevant for real-time disease diagnosis. The microscope design allows for many different microscopic imaging modalities to be readily implemented, making it a powerful tool for many different clinical contexts.

\section{Acknowledgments}

We thank F. van Gaal (VDL), C. van der Vleuten, and R. van Rijswijk (MiPlaza) for their technical support and G. Braun and R. Harbers (Philips Research) for their experimental support. M. van der Mark and J. Schleipen (Philips Research) provided valuable feedback in the manuscript preparation phase. We thank M. van Zandvoort and R. Megens (Maastricht University) for their insights on the cardiovascular images.

\section{References}

1. P. Delaney and M. Harris, Chapter 26 in Handbook of Biological Confocal Microscopy, J. B. Pawley, Ed., Springer, New York (2006).

2. A. D. Mehta, J. C. Jung, B. A. Flusberg, and M. J. Schnitzer, "Fiber optic in vivo imaging in the mammalian nervous system," Curr. Opin. Neurobiol. 14(5), 617-628 (2004).

3. B. A. Flusberg, E. D. Cocker, W. Piyawattanametha, J. C. Jung, E. L. M. Cheung, and M. J. Schnitzer, "Fiber-optic fluorescence imaging," Nat. Methods 2(12), 941-950 (2005).

4. E. Laemmel, M. Genet, G. Le Goualher, A. Perchant, J-F. Le Gargasson, and E. Vicaut, "Fibered confocal fluorescence microscopy $\left(\right.$ Cell-viZio $\left.{ }^{\mathrm{TM}}\right)$ facilitates extended imaging in the field of microcirculation," J. Vasc. Res. 41(5), 400-411 (2004).

5. B. A. Flusberg, A. Nimmerjahn, E. D. Cocker, E. A. Mukamel, R. P. J. Barretto, T. H. Ko, L. D. Burns, J. C. Jung, and M. J. Schnitzer, "High-speed, miniaturized fluorescence microscopy in freely moving mice," Nat. Methods 5(11), 935-938 (2008).

6. J. Sun, C. Shu, B. Appiah, and R. Drezek, "Needle-compatible single fiber bundle image guide reflectance endoscope," J. Biomed. Opt. 15(4), 040502 (2010).
7. T. Ota, "In situ fluorescence imaging of organs through compact scanning head for confocal laser microscopy," J. Biomed. Opt. 10(2), 024010 (2005).

8. C. J. Engelbrecht, R. S. Johnton, E. J. Seibel, and F. Helmchen, "Ultra-compact fiber-optic two-photon miscroscope for functional fluorescence imaging in vivo," Opt. Express 16(8), 5556-5564 (2008).

9. H-J. Shin, M. KC. Pierce, D. Lee, H. Ra, O. Solgaard, and R. RichardsKortum, "Fiber-optic confocal miscroscope using MEMS scanner and miniature objective lens," Opt. Express 15(15), 9113 (2007).

10. J. T. C. Liu, M. J. Mandella, H. Ra, L. K. Wong, O. Solgaard, G. S. Kino, W. Piyawattanametha, C. H. Contag, and T. D. Wang, "Miniature near-infrared dual-axes confocal microscope utilizing a twodimensional microelectromechanical systems scanner," Opt. Lett. 32(3), 256-258 (2007)

11. P. Delaney, M. Harris, and R. G. King, "Fiber-optic laser scanning confocal microscope suitable for fluorescence imaging," Appl. Opt. 33(4), 573-577 (1994).

12. M. D. Chidley, K. D. Carlson, R. R. Richards-Kortum, and M. R. Descour, "Design, assembly, and optical bench testing of a high-numericalaperture miniature injection-molded objective for fiber-optic confocal reflectance spectroscopy," Appl. Opt. 45(11), 2545-2554 (2006).

13. K. Carlson, M. Chidley, K-B. Sung, M. Descour, A. Gillenmwater, M. Follen, and R. Richards-Kortum, "In vivo fiber-optic confocal reflectance microscope with an injection-molded plastic miniature objective lens," Appl. Opt. 44(10), 1792-1797 (2005).

14. J. C. Jung, A. D. Mehta, E. Aksay, R. Stepnoski, and M. J. Schnitzer, "In vivo mammalian brain imaging using one- and two-photon fluorescence microendoscopy," J. Neurophysiol. 92(5), 3121-3133 (2004).

15. J. C. Jung and M. J. Schnitzer, "Multiphoton endoscopy," Opt. Lett. 28(11), 902-904 (2003)

16. M. T. Myaing, D. J. MacDonald, and X. Li, "Fiber-optic scanning two-photon fluorescence endosocope," Opt. Lett. 31(8), 1076-1078 (2006).

17. G. J. Tearney, M. E. Brezinski, B. E. Bouma, S. A. Boppart, C. Pitris, J. F. Southern, and J. G. Fujimoto, "In vivo endoscopic optical biopsy with optical coherence tomography," Science 276(5321), 2037-2039 (1997).

18. X. Li, C. Chudoba, T. Ko, C. Pitris, and J. G. Fujimoto, "Imaging needle for optical coherence tomography," Opt. Lett. 25(20), 15201522 (2000).

19. M. Kanai, "Condensing optical system, confocal optical system and scanning confocal endoscope," U.S. Patent No. 7338439 (2008).

20. B. H. W. Hendriks, M. A. J. van As, and P. J. H. Bloemen, "Miniaturisation of high-NA objectives for optical recording," Opt. Rev. 10(4), 241-245 (2003)

21. I. Walmsley, L. Waxer, and C. Dorrer, "The role of dispersion in ultrafast optics," Rev. Sci. Instrum. 72(1), 1-29 (2001).

22. Z. Bor, "Distortion of femtosecond laser pulses in lenses and lens systems," J. Mod. Opt. 35(12), 1907-1918 (1988).

23. J. M. Khosrofian and B. A. Garetz, "Measurement of a Gaussian laser beam diameter through the direct inversion of knife-edge data," Appl. Opt. 22(21), 3406-3410 (1983)

24. M. Rajadhyaksha, R. R. Anderson, and R. H. Webb, "Video-rate confocal scanning laser microscope for imaging human tissue in vivo," Appl. Opt. 38(10), 2105-2115 (1999).

25. Richardson Technologies Inc., T. M. Richardson, "Test slide for microscopes and method for the production of such slide," U. S. Patent No. 6381013 (2002), www.emsdiasum.com.

26. R. T. A. Megens, M. A. M. J. van Zandvoort, M. G. A. oude Egbrink, M. Merkx, and D. W. Slaaf, "Two-photon microcopy on vital arteries: imaging the relationship between collagen and inflammatory cells in atherosderotic plagues," J. Biomed. Opt. 13(4), 044022 (2008). 